leach out of the clay. Very low level radioactive clay could simply be buried."

Komarneni and co-researchers Naofumi Kozai, a visiting scientist from the Japan Atomic Energy Research Institute, and William J. Paulus, master's degree recipient, now at General Motors Corporation, found that if the mica is only partially filled with radium at the time of disposal, then heating to above $100^{\circ} \mathrm{C}$ will lock the radium in place.

Sodium -4 mica, the researchers found, is easily synthesized by heating kaolinitea naturally occurring clay with an equal ratio of silicon and aluminum-with magnesium oxide and sodium fluoride to about $815^{\circ} \mathrm{C}$.

Sodium- 4 mica could be used in conventional ion-exchange columns to remove radium from water, but would first need to be pelletized. To immobilize radium from mine or mill tailings, the researchers said that mixing the clay with the tailings is sufficient. They also suggested that the clay could be used to line ponds that receive radium-containing tailing water to prevent migration from the pond, or clay curtains can be placed around tailings to keep the radium inside.

\section{Linear Defects Stabilize Magnetic Domain Walls}

Ultrathin cobalt films or multilayered structures, magnetized perpendicular to the thin-film planes, have greatly increased storage densities by solving the thermal "flips" (i.e., magnetic spin reversals) problem that leads to the loss of stored information. However, extension of this technique to storage densities larger than 1 Tbit in. $^{-2}$ suffers from the roughness and mobility of the magnetic domain walls (DWs), which prohibits closer packing of the storage bits. In order to overcome this problem and achieve even higher storage density, researchers from Los Alamos National Laboratory and IBM T.J. Watson and Almaden Research Centers have utilized long-range strain fields caused by introduced linear defects.

When ultrathin trilayers (sandwich structures $)$ of $\operatorname{Pt}(3 \mathrm{~nm}$, top $) / \mathrm{Co}(0.7$ $\mathrm{nm}) / \mathrm{Pt}(2 \mathrm{~nm}$, bottom) are deposited, the domains exist in round shape with rugged walls. The same problem occurs in pattern nucleation sites. The velocity of DWs increases swiftly when the magnetic field is beyond $H_{\mathrm{c}}=750$ Oe (the velocity

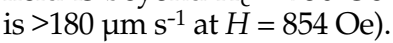

Noting examples that column defects can strongly localize wandering vortex lines in high-temperature superconductors, the researchers installed a line defect in the cobalt layer by clamping the substrate to produce anisotropic tension during the cobalt deposition, and then releasing the sample. As reported in the March 22 issue of Nature, the introduced $y$-axis-invariant strain field $\varepsilon(x)$ influences the behavior of domains through magnetoelastic coupling. First, it accommodates the walls along the linear defect. Second, by increasing the elasticity, it reduces the wall roughness. Finally, it decelerates the motion of DWs to a nearly full stop when the field is high enough.

Polar magneto-optic Kerr microscopy images show that the conformance of the DWs to the defect line occurs even at a large distance $(\sim 300 \mu \mathrm{m})$. The closer the DWs come to the defects, the smoother (straighter) they are. The deceleration rate also depends on the proximity to the defects. The researchers found a nonlinear relationship of $v-H$ curve (where $v-H$ refers to voltage-versus-field), which is regarded as a sign of glassy (creep) dynamics. A higher field $H$ is needed to get closer to the line defects, but after a

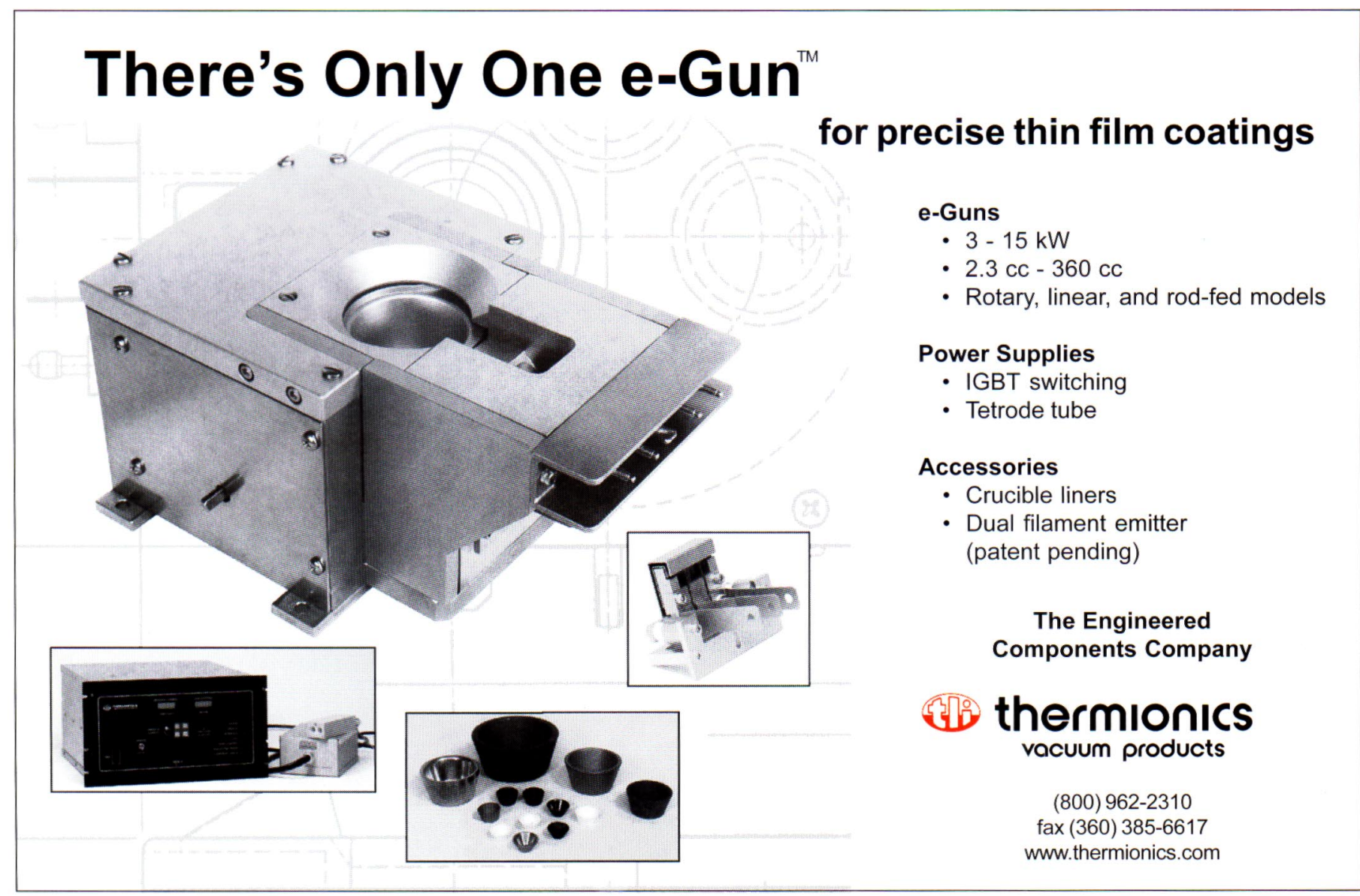

Circle No. 5 on Inside Back Cover 
sufficient time period ( $t=1800 \mathrm{~s})$, two DWs approaching the defects from each side are effectively stationary.

The line defects act as "chalk lines" to straighten the DWs in the magnetic recording layers. Only a few defects stabilize large areas of films. Good results were produced in field up to $\sim 2 \mathrm{H}_{\mathrm{c}}$. The researchers said that situations at higher fields $\left(H \sim 32 H_{\mathrm{c}}\right)$ and speeds of current recording technology $\left(1 \mathrm{Gbit} \mathrm{s}^{-1}\right)$ need further studies.

\section{Li ZENG}

\section{Spinodal Decomposition Underlies Evolution of Nanoporosity in De-Alloying}

The selective dissolution of an alloy is a well-known de-alloying process with broad applications in the chemical industry. During the de-alloying process, the less noble elements of the alloy are dissolved while the more noble elements remain. After selective dissolution, a nanoporous sponge is formed by the remaining noble elements.

Although the chemical phenomenon is well documented, the physical mechanism of selective dissolution has remained unclear. A team of researchers from Johns Hopkins, Harvard, Northeastern, and Arizona State universities have developed a scenario for the de-alloying process, reporting in the March 22 issue of Nature that the effect known as spinodal decomposition is responsible for the characteristic nanoporosity size. Jonah Erlebacher of Johns Hopkins and his research team used critical potential as an index to construct a simulation model.

Basing their experiments on the dealloying of Ag-Au alloy, Erlebacher and his research team placed the alloys into an electrolyte and measured the critical potential of the alloys with different alloy compositions. After the dissolution, scanning electron micrographs showed a nanoporous sponge composed of gold on the surface of the alloy with ligament spacings of the order of $10 \mathrm{~nm}$.

The researchers proposed the following scenario. Initially, a single silver atom on a de-alloying surface is dissolved. Then, the nearby atoms are more easily dissolved because of the vacancy. But as a more noble element, the gold atoms on the surface do not dissolve. They agglomerate by diffusion into "islands." At first, the tops of the "islands" are gold-rich while the bases are not de-alloyed material. As more and more silver atoms are dissolved, the bases of the islands become exposed to the electrolyte.

"These mounds get undercut, increasing the surface area that gold must cover to bring about passivation," according to the researchers. So, by agglomerating together while keeping enough surface area, the gold atoms evolve into a nanoporous sponge.

When the nanoporosity size is large, the sponge will grow slowly because of the time required for gold to diffuse. On the other hand, the sponge will also grow slowly if the nanoporosity size is small because, the researchers reported, "shortlength-scale fluctuations create much energetically unfavorable incipient interface between phases, inhibiting their growth." This effect, known as spinodal decomposition, is the reason why the nanoporosity can have a characteristic spacing.

Based on their observations and critical potentials measurement, Erlebacher and his team developed a Monte Carlo model on the (111) alloy surface, covering the diffusion of silver and gold and the dissolution of silver, to simulate the de-alloying. They report that their model is consistent with both the predictions based on continuum theory and their experimental data. Compared with the well-known results of the vapor-phase deposition case, they said, their results do not depend on the reversibility of the aggregation, which suggests the existence of universal scaling laws for aggregation.

\section{Cost-Effective Portable Spin Goaters

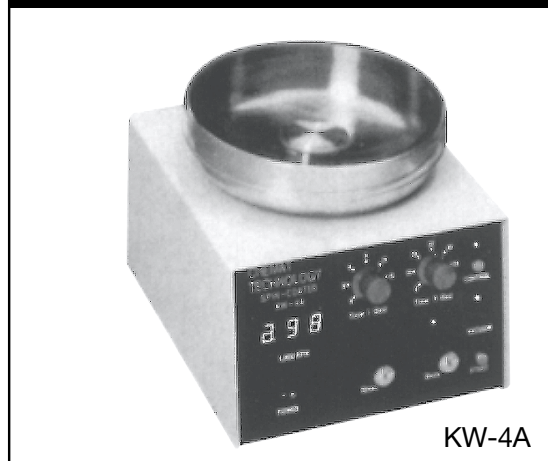 \\ Two-Stage Spinning \\ - Dispense liquid during Stage 1 \\ - Spin-up and flatten during Stage 2 \\ Adjustable Speed \\ Stage 1 \\ - 500 to $2,500 \mathrm{rpm}$ \\ - 2 to 18 seconds \\ Stage 2 \\ - 1,000 to $8,000 \mathrm{rpm}$ \\ - 3 to 60 seconds \\ Precision Video Biological Microscope}

\section{Only \$1,968.90 \\ (including Video Camera)}

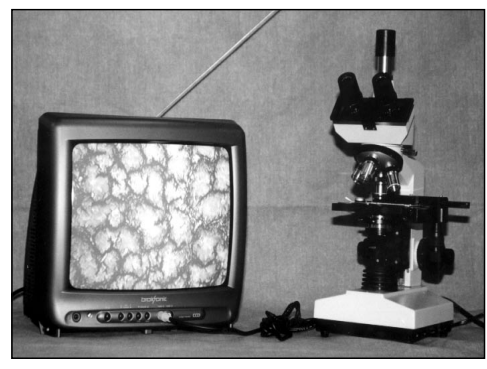

XSZ-107CCD

\section{Features}

- Fully Coated Optical System

- 45mm Achromatic Objective, Parfocall

- Coaxial Coarse and Fine Focus Adjustment

- Focusing Stops to prevent Objectives \& Slides from being Damaged

- Built-in Illumination, Adjustable

- Brightness

\section{Specifications}

- Trinocular Head for Video Camera

- Wide Field Eyepieces WF 10X, P16X( WF 16X)

- Achromatic 4X, 10X, 40X(S) and $100 X(S$, Oil)

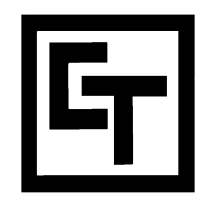

\section{CHEMAT TECHNOLOGY, INC.}

9036 Winnetka Avenue, Northridge, CA 91324

U.S. Toll-Free 800-475-3628 • Non-U.S. 818-727-9786

Fax: 818-727-9477 • E-mail: chemat@aol.com www.chemat.com 